\title{
Designing Effective Soldier-Robot Teams in Complex Environments: Training, Interfaces, and Individual Differences
}

\author{
Michael J. Barnes ${ }^{1}$, Jessie Y.C. Chen ${ }^{1}$, Florian Jentsch ${ }^{2}$, and Elizabeth S. Redden ${ }^{1}$ \\ ${ }^{1}$ U.S. Army Research Laboratory - Human Research \& Engineering Directorate \\ Bldg 459, Aberdeen Proving Ground, MD 21005, USA \\ ${ }^{2}$ University of Central Florida - Department of Psychology \\ Orlando, FL 32816, USA \\ \{michael.j.barnes, jessie.chen, elizabeth.redden\}@us.army.mil, \\ fjentsch@mail.ucf.edu
}

\begin{abstract}
Extensive US Army programs are being pursued to increase the effectiveness of unmanned vehicles for diverse missions during future combat. The following paper identified 23 human-robot interaction (HRI) guidelines related to interface design, procedural issues, individual differences and training implications based on three HRI research programs. The programs range from simulation experiments that investigated robot control in a multitasking environment from a mounted combat vehicle, to reconnaissance missions in a miniature Iraqi city that focused on Soldier-robot teaming relationships, to field studies at Ft. Benning that examined interface design issues for Soldiers supervising or controlling small robots.
\end{abstract}

Keywords: HRI design, military, human factors.

\section{Introduction}

The U.S. Army is engaged in an extensive research program whose goal is to develop unmanned vehicles (UVs) including both ground (UGV) and aerial versions (UAS) to act as force multipliers, to increase tactical flexibility, and most important, to save Soldiers' lives. While the eventual goal is autonomy, the current and near-term systems require teleoperations or waypoint control making the Soldier responsible for controlling the UVs and adapting to change. But even more important than control is the Soldier's role in understanding the mission environment both in terms of specific goals and meta-goals. For example, Robin Murphy [1] in reviewing real-world experiences with robotic systems points out that situation awareness (SA) rather than teleoperations was the most difficult problem for operators to overcome in finding survivors during the World Trade Center disaster. The purpose of this paper is to review three research programs funded by the Army in order to derive general principles and guidelines. These three programs were chosen because of their diverse approaches as well as their extensive findings over six or more years of research. All the programs were realistic in the sense that they dealt with real Army problems in 
operational settings. For each effort, we evaluated the implications of their results and derived general guidelines/ principles for designing UV systems that enhance Soldier HRI performance in diverse mission environments. We briefly describe the three paradigms, their most important results as well as guidelines for interface design, Soldier procedures, required skill sets, and training implications.

\section{Simulation Studies at ARL-Orlando}

The simulations conducted in Orlando were designed to investigate operator control or supervision of robots from a mounted vehicle during multitasking mission segments. The first four experiments emphasized difficulties mounted crews encountered when performing their normal duties in addition to remote targeting with a robot. The simulation studies manipulated both multi-tasking variables and degree of robotic control using a OneSAF simulation of a maneuver scenario in which the operator had to detect targets near the manned vehicles (primary task) and remote targets viewed from the robot's video (secondary task) as well as a communications and SA tasks. The initial experiment examined span of control issues whereas the latter three experiments manipulated variables related to the gunner conducting the robotic and gunner functions using separate displays. More important from a theoretical point of view was the impact of individual differences related to perceived attentional control (PAC) and spatial ability (SpA). Two of the experiments manipulated mitigation factors such as tactile cueing, aided target recognition (AiTR), and reliability - including both miss prone and false alarm prone AiTRs.

Three later experiments investigated the use of an intelligent agent, RoboLeader, to control 4-8 robots allowing the operator to supervise the agent instead of being required to control individual robots. The RoboLeader experiments were simulated in the MIX Testbed with an emulated operator control unit showing both map and streaming video views from the individual robots. Variables manipulated included number of robots, agent vs. direct control, individual differences, workload, SA, target density, and reliability (miss- and false alarm- prone).

\subsection{Summary of Results}

The initial mounted study indicated that operators were not efficient in using more than a single asset concurrently. They tended to over-rely on UASs compared to semiautonomous UGVs although performance was approximately the same under single asset conditions [2]. In the gunner's study, operators performed more poorly on both the primary local security task and the secondary robotic remote targeting task as a function of robotic task difficulty [3]. In the third experiment, AiTR improved targeting on the primary task but had mixed results on the robotic task. Contrary to previous research, participants had better robotics task performance when they teleoperated the robot than when the robot was semi autonomous - suggesting overreliance on automation for robotic tasks although the participants were aware of the limitations of the AiTR system on the robots [4]. In general, the experiments showed pronounced individual differences with participants with low SpA abilities and low PAC scores performing more poorly than those with better scores. However, there 
were some interesting interactions; the AiTR was more beneficial to the low SpA participants raising targeting accuracy nearly to those levels of the more proficient SpA participants; there was a similar trend for low PAC participants [4]. Also, low SpA participants tended to prefer visual cueing whereas high SpA participants were satisfied with tactile cueing. The PAC interactions were even more pronounced in the AiTR reliability study [5]; high PACs tended to perform poorly when using falsealarm-prone aids whereas low PACs tended to perform more poorly on the missprone aids showing a classical type $\mathrm{X}$ interaction indicating possible mistrust in the former case and over trust in the latter case. The RoboLeader studies investigated the usefulness of an intelligent agent to help supervise multiple robots [6][7]. RoboLeader supervision vs. operator supervision did not result in improved target detection for both the 4 and 8 robot conditions but RoboLeader supervision did result in more rapid transit for the robots. [6]. In the second intelligent agent experiment, the type of unreliability of the RoboLeader's route change suggestions had unexpected results [7]. The false-alarm-prone conditions (suggesting new routes when it was not necessary) actually resulted in better performance than the conditions with miss-prone suggestions (RoboLeader failed to suggest a new route when it was appropriate) because the false-alarm-prone suggestions could be easily checked by viewing the map but missing suggestions were more difficult to verify. Again, individual differences were important; high SpA participants performed better in scanning and target detection whereas high PAC participants' showed superior performance in the secondary tasks - communications and gauge monitoring [7].

\subsection{Guidelines}

1. Teleoperation creates significantly more workload for the operators than does other robotics tasks. In multitasking environments, operators may overlook other concurrent tasks or overly rely on automation for other tasks when teleoperating a robot. Although many of the ground robotic assets in the future will be semiautonomous, teleoperation will still be an important part of any missions involving robotic assets (e.g., when robots encounter obstacles or other problems). The higher workload associated with teleoperation needs to be taken into account when designing the user interfaces for the robotic assets (see [8] for potential user interface designs).

2. Unreliable automated systems tend to affect operator's performance of concurrent tasks involving visual scanning and his/her situation awareness of the overall tasking environment [7] due to the operator's having to constantly monitor the automated system. Cueing displays in conjunction with aided target recognition capabilities should be implemented to enhance operator's overall task performance.

3. Participants' spatial ability ( $\mathrm{SpA}$ ) was found to be a reliable predictor of their targeting task performance. When selecting personnel for operating robotics systems for reconnaissance tasks, operators' SpA should be one of the criteria under consideration. This is consistent with conclusions of recent U.S. Air Force studies on the required abilities of UAS pilots and sensor operators [9].

4. The data from the studies conducted by Chen and her colleagues showed that those with lower SpA tend to prefer visual cueing over tactile cueing (in a visually intensive, multitasking environment), and those with higher SpA tend to favor 
tactile cueing over visual cueing. To better enhance the task performance for low SpA individuals, the visual cueing display should be more integrated with the visual scene. For example, augmented reality (i.e., visual overlays) is a potential technique to embed directional information onto the video.

5. Operators' attentional control ability appears to have an impact on their reliance on automation and responses to unreliable alerts, especially when workload is heavy (e.g., teleoperating a robot while multitasking). Lee and See [10] suggested that training can be developed to educate the operators regarding the alert's "expected reliability, the mechanisms governing its behavior, and its intended use" (p. 74).

6. When selecting personnel for positions with frequent multitasking demands, attentional control abilities should be considered. A recent U.S. Air Force study concluded that attention control is one of the critical abilities of UAS pilots [9]. Consistent with this finding, the results in communication task performance in the studies conducted by Chen and her colleagues showed that those with better attentional control consistently outperformed those with lower attentional control. If personnel selection is not feasible, then training programs should be developed to improve the attention management skills of the low PAC operators to support multitasking requirements.

\section{Human-Robot Teaming Research at Univ. of Central Florida}

The research conducted at University of Central Florida (UCF) focused on the performance of teams of multiple operators as they interacted with one or more robotic vehicles. In all studies, of which there were over 12 between 2004 and the end of 2010, the teams' task was to perform Reconnaissance, Surveillance, and Target Acquisition (RSTA) in a mixed urban/suburban environment, represented by a custom-built 1:35 toy city prototypical of urban environments in Iraq [11]. The robotic assets in the simulation were UGVs that in size and mission resembled the Army's planned Armed Robotic Vehicle (ARV) (i.e., a vehicle of roughly the size of a small truck or small armored fighting vehicle). They were operated by confederate experimenters in a "man-behind-the-curtain" fashion; that is, the vehicles were operated by the confederates according to a script that specified the level of autonomy (LOA) while the research participants believed that the vehicles were real robots. Additionally, several studies employed UASs, which were simulated by planar and oblique camera(s) moving above the toy village in patterns similar to reconnaissance patterns employed by real UASs.

The conceptual frameworks underlying the research were (a) the Team Effectiveness Model (TEM), an input-process-output model of team processes, and (b) a conceptualization that put team performance of the mixed human-robot team at the top of a hierarchy with three underlying, or enabling, topics necessary to achieve team performance. The three underlying topics were: (a) Mutual planning and shared mental models in teams; (b) Self-localization and SA while using robotic/unmanned vehicles; and (c) Target recognition, friend-foe discrimination, and vehicle identification/ classification. Only when the teams were able to correctly and efficiently perform all three underlying tasks, we posited, would they be able to engage in successful team 
performance. Different tracks within the research program, therefore, investigated the underlying topics and their determinants.

\subsection{Summary of Results}

Although the number of studies precludes a discussion of all results obtained through this research program, a number of highlights stood out in each area. First, conducting RSTA missions with UVs is an inherently difficult task; even well-trained teams with a military background found it both effortful and difficult to monitor the vehicles' paths, maintain spatial and situation awareness, detect and identify targets, and report them, while monitoring system health, reacting to unforeseen situations, etc. Further, precisely because the RSTA task is an attention- and memory-intensive task, we found that the addition of team members was helpful and generally improved performance, regardless of whether we changed from individual operators to teams of two, or from a team of two operators to a recon team with two operators and one mission manager. Indeed, under difficult circumstances and high workload, the addition of a team member improved performance by more than 1 person unit. In one study, the addition of a team member to an individual operator, thereby creating a team of two operators, improved performance by $190 \%$. Clearly, the RSTA task is, at least at the current LOA, resource-intensive enough that the gains in performance from the addition of team members far outweigh the process losses typically associated with the communication and coordination necessary for team performance.

Our studies, however, did provide a more detailed picture than the mere statement that "adding team members is better." These studies also showed that technical, procedural, and individual factors all impacted performance, in cases to a degree that offset the addition of team members. For example, when we studied whether adding a third team member as a supervisor improved team performance, we found that the same performance gains as engendered by the addition of the team member could be observed when the original UGV operator in a UGV-UAS team had very high spatial abilities [12]. In this case, the team member with the high spatial abilities could lead the team to great performance, and the addition of a third team member added little in performance. Similarly, team performance shifted by amounts similar to those related to the addition of a team member when we changed the type of communications and interactions that the operator team could perform. For example, allowing teams of two operators of dissimilar vehicles to share imagery significantly and substantially improved their performance in RSTA tasks over solely being allowed to communicate verbally or via text. Allowing the team members to share not only imagery, but also control over their partner's reconnaissance payload, however, was associated with reductions in performance as the team members got in each other's way and unfamiliarity with the other systems sensors made individual performance poorer [13].

Another variable that interacted with the team members' individual abilities, as well as with the technical and procedural solutions they were given to use, was the LOA and automation of the UVs. In a replication of a previous study, we found that different aspects of the RSTA task benefited from different levels of UV autonomy. For example, with respect to situation and spatial awareness of the operator(s), we found that a middle LOA, similar to supervisory control in automation, was most beneficial. In this case, operators had the highest spatial awareness when the system 
could traverse point-to-point without needing constant operator intervention (i.e., did not require teleoperation), but required the operator to actively select from systemgenerated suggestions and options for the next waypoint, rather than executing the movement fully autonomously. Conversely, when the task that was automated required substantial interaction with the system(s), such as inputting commands and waypoints to two vehicles so that they could execute a joint task, a higher LOA that addressed those specific tasks was helpful and associated with better performance.

Finally, the series of studies clearly demonstrated that our conceptualization of the three components of performance for successful human-robot teams was correct. Understanding the RSTA performance of teams of multiple operators interacting with multiple UVs, required an understanding of (a) planning and shared understanding of the team members, (b) spatial and situation awareness, and (c) target recognition and identification. When teams were constrained or deficient in any of the three underlying aspects, their team performance would suffer. Also, attempts to improve the performance of teams would have to target specific variables influencing performance on the underlying topics. For example, spatial abilities of the team members influenced not only spatial and situation awareness but also mission planning and ultimately team performance. Consequently, it is important to assess the impact of a system change or intervention not only on the most closely related underlying aspect, but also the others, when one wants to improve overall team performance.

\subsection{Guidelines}

1. At this point, operator-to-vehicle ratios of less than 1:1 are unrealistic in RSTA tasks. Although a single operator may be able to control more than one unmanned system in other tasks, such as logistics ("mule-train") or casualty evacuation, this is not the case for RSTA tasks where creating small teams of operators that interact at 1:1 operator-to-vehicle ratios or higher is beneficial.

2. LOAs should be chosen to match the appropriate task aspect. For spatial awareness, neither manual control (i.e., teleoperation) nor full autonomy are desirable; the former requires too many operator cognitive resources, whereas the latter leaves the operator "out-of-the-loop" and therefore unaware of system status and location.

3. Promising targets for automation are task aspects that require high levels of operator involvement and particularly "busywork." Path planning, for example, is best supported by automation that simplifies the entry of coordinates (e.g., from keystroke entry to map point-and-click) and combines the different task demands in one, integrated display.

4. The unique and frequently unfamiliar views from robot-mounted displays are difficult to interpret for human operators (e.g., "soda straw effect"). This makes target location and identification very difficult. Automation and structured training are needed if operators are not to spend long hours in inefficient on-the-job training.

5. The impact of technical and procedural interventions/changes to the UV system needs to be considered at multiple levels. An intervention that facilitates path planning but lowers SA may do more harm than good. Conversely, providing the 
operators with integrated displays that show obstacles, own forces, likely threats, etc. may improve planning, SA, and target detection simultaneously.

6. Sharing information among team members is typically positive; it allows them to build shared mental models and reduces the need for communications to identify and localize features in the environment. Allowing team members to share control of vehicles, however, is typically not beneficial as it interferes with the team members' individual tasks and can lead to confusion.

7. The spatial ability of individual operators is a powerful predictor of individual and team performance in human-robot teams. In some situations, one team member with high spatial abilities can make up for the addition of a team member that would otherwise coordinate the actions of the team.

\section{Field Studies at ARL-Ft. Benning}

The purpose of the research program at Ft. Benning was to develop intuitive interfaces for the dismounted infantry to control small UGVs (SUGVs) in a realistic environment. Ft Benning, Georgia was ideal because of the supply of experienced infantry Soldiers who gave the experimenters valuable feedback as well performance data expected from target audience Soldiers. The initial efforts involved investigating scalable displays concepts to find the best solutions for a display in terms of size and performance. Subsequent studies investigated controls, multimodal interfaces, gogglemounted displays, voice controls, LOA, telepresence and the use of cell phones to control the robot. The purpose of the latter studies was to understand th effects of progressive autonomy on Soldier effectiveness and SA. In general, the procedures were similar, with the Soldiers being in ether a mobile or stationary control condition. The multivariate performance data included deviations from the course, time to complete the course, target detection and latency [14].

\subsection{Summary of Results}

The initial study determined that the operator could drive TALON SUGVs effectively with 3.5 inch and 6.5 PDA sized displays [15]. Goggle-mounted displays caused perceptual problems during the experiment without compensatory performance gains in three experiments [15][16]. It is important to note that dismounted operations will require map and other information for the Soldiers to navigate effectively beyond line of sight in complex terrain. In the next study [17], Redden and colleagues examined three options to combine map and video control: 1) toggle between two 3.5-inch displays, 2) use a 6.5-inch split screen display, or 3) use a 3.5-inch toggle display with a tactile belt to replace the visual map display. They found that navigation with a 3.5-inch display with tactile augmentation performed as well as the 6.5-inch split screen display indicating that a tactile belt was an efficient means of displaying map information while reducing display real estate. In contrast, when Soldiers were required to toggle back and forth between the two 3.5-inch displays, their performance proved to be less effective [18]. Other studies investigated control options for reducing control surfaces [19][20]. The most efficient way to reduce controller size for discrete functions was a voice activated system permitting hands-free operations, an extremely important advantage 
for the infantry; however, voice control had limited effectiveness when commands required a continuous motion such as turn left [20]. Reducing control size by miniaturizing controls was preferable to reducing the number of controls by creating more multifunction controls [19]. Recent research investigated more advanced systems developed by ARL, TNO laboratories in the Netherlands, and the SPAWAR Naval Laboratory. The ARL experiment investigated the use of the Android size (about 2.5 inches) cell phone to control semi-autonomous PACBOTs resulting in limited success because of lack of control responsiveness [21]. The TNO telepresence experiments showed that adding auditory 3-D augmentation along with a head-track camera control improved robotic navigation time and target identification for audio-based localization tasks, compared to visual displays [22]. The Navy researchers at SPAWAR used a Multiple Operations Control Unit (MOCU) to monitor autonomous and semiautonomous SUGVs which were compared to teleoperated systems. Participants performed significantly more rapidly and were less error prone for driving performance using the MOCU interface to monitor the autonomous and semi-autonomous robots compared to teleoperated conditions while their ability to find targets was not affected by LOA [23].

\subsection{Guidelines and Lessons Learned}

1. Displays as small as 3.5 and 6.5 inches can be used for camera-based teleoperation and local surveillance with small, slow-speed robots and displays as small as 4.3 inches are possible for viewing of video from remote UGVs and UASs.

2. Monocular helmet -mounted displays (HMDs) for robots can be problematic and should be carefully assessed before use to reduce the chance that binocular rivalry, over-stimulation, excessive head borne weight and loss of local SA. They also appear problematic for passive viewing, especially when a controller such as a mouse must be used to complete a task.

3. Speech-based control shows promise for robotic operation when commands are intuitive and based upon the target population word use but not for all functions. They are easy to use and show the potential to increase secondary task performance. They have provided decreased time and effort when performing simultaneous tasks in conjunction with manual controls. Continuous control commands (e.g., pan, drive in a circle) are more efficiently controlled manually.

4. Our results show the efficacy of a tactile belt to augment robotic OCUs to reduce required display size for navigation beyond line of sight.

5. Reducing a controller size by shrinking the size of the individual controls had less adverse impact on performance than reducing its size by reducing the number of controls.

6. As levels of autonomy increase, workload, reconnaissance times, and driving errors decrease and accuracy of mental maps increase.

7. Soldiers prefer the bounding technique (moving and stopping to control the SUGV) to the continuous movement technique when moving with robots. Fewer driving and off course errors were made and more items were detected when the bounding movement was used than when the continuous movement was used. Until robots become more autonomous in their navigation, robotic control during Soldier movement is beyond the multitasking ability of most Soldiers. As reliable 
autonomy in robots increases, Soldiers should have more available free time to divert their attention away from the robot and toward their own environment, enabling side-by-side movement.

8. Soldiers with higher video gaming skill appear to be more proficient with the hand and eye component of teleoperations.

9. Visual attendance on robotic displays should not be wider than the 5 degree macular vision (the actual width depends upon the viewing distance) in order to avoid visual scanning which can result in fatigue and missed data.

10. Head-track cameras and telepresence sensors were associated with faster, easier performance, and were preferred by Soldiers over baseline joystick controls with mono vision and audio. However, there is still a challenge with telepresence because of motion sickness experienced by some operators.

\section{Conclusions}

As Soldiers become more involved in supervising and controlling UVs, they will face multiple challenges related to multi-tasking, poor interface design, manual control, automation reliability, deficient skill sets, and poor SA. Twenty-three specific guidelines to overcome these barriers were derived from three diverse lines of HRI research. The guidelines included criteria for: (a) selection and training of Soldiers that are sensitive to individual differences in attentional control and spatial reasoning, (b) automated decision support, (c) use of intelligent agents, (d) concepts to improve teaming and shared mental models, (e) multimodal and scalable interfaces. Furthermore, the results will help establish a framework for the development of progressive autonomy strategies to reduce operator's workload while improving SA.

\section{References}

1. Murphy, R.: Human-Robot Interactions in Rescue Robots. IEEE Trans. Sys., Man \& Cybern. 34, 1-15 (2004)

2. Chen, J.Y.C., Durlach, P., Sloan, J., Bowens, L.: Human Robot Interaction in the Context of Simulated Route Reconnaissance Missions. Military Psychology 20, 135-149 (2008)

3. Chen, J.Y.C., Joyner, C.T.: Concurrent Performance of Gunner's and Robotics Operator's Tasks in a Multi-Tasking Environment. Military Psychology 21, 98-113 (2009)

4. Chen, J.Y.C., Terrence, P.I.: Effects of Tactile Cueing on Concurrent Performance of Military and Robotics Tasks in a Simulated Multi-Tasking Environment. Ergonomics 51, 1137-1152 (2008)

5. Chen, J.Y.C., Terrence, P.I.: Effects of Imperfect Automation on Concurrent Performance of Military and Robotics Tasks in a Simulated Multi-Tasking Environment. Ergonomic 52, 907-920 (2009)

6. Chen, J.Y.C., Barnes, M.J.: Supervisory Control of Robots Using RoboLeader. In: Human Factors \& Ergo. Soc. 54 ${ }^{\text {th }}$ Annu. Mtg, pp. 1483-1487. HFES, Santa Monica (2010)

7. Chen, J.Y.C., Barnes, M.J., Kenny, C.: Effects of Unreliable Automation and Individual Differences on Operator's Supervisory Control of Multiple Ground Robots. In: $6^{\text {th }}$ ACM/IEEE International Conference on Human-Robot Interaction. ACM, New York (2011) 
8. Chen, J.Y.C., Haas, E.C., Barnes, M.J.: Human Performance Issues and User Interface Design for Teleoperated Robots. IEEE Transactions on Systems, Man, and CyberneticsPart C: Applications and Reviews 37, 1231-1245 (2007)

9. Chappelle, W.L., McMillan, K.K., Novy, P.L., McDonald, K.: Psychological Profile of USAF Unmanned Aerial Systems Predator \& Reaper Pilots. Aviation, Space, and Environmental Medicine 81, 339 (2010)

10. Lee, J., See, K.: Trust in Automation: Designing for Appropriate Reliance. Human Factors 46, 50-80 (2004)

11. Jentsch, F., Evans, A., Ososky, S.: Military HRI Research Conducted Using a Scale MOUT Facility. In: Barnes, M., Jentsch, F. (eds.) Human-Robot Interactions in Future Military Operations, Ashgate, Hampshire, UK, pp. 419-431 (2010)

12. Fincannon, T., Evans, A., Phillips, E., Jentsch, F., Keebler, J.: The Influence of Team Size and Communication Modality on Team Effectiveness with Unmanned Systems. In: Human Factors \& Ergo. Soc. 53 ${ }^{\text {rd }}$ Annu. Mtg, pp. 419-423. HFES, Santa Monica (2009)

13. Fincannon, T., Keebler, J., Jentsch, F., Phillips, E., Evans, A.: Team Size, Team Role, Communication Modality, and Team Coordination in the Distributed Operation of Multiple Heterogeneous Unmanned Vehicles. J. Cog. Eng. \& Decision Making (Special Issue on Improving Human-Robot Interaction) (in press)

14. Redden, E.S., Elliott, L.R.: Robotic Control Systems for Dismounted Soldiers. In: Barnes, M., Jentsch, F. (eds.) Human-Robot Interaction in Future Military Operations, Ashgate, Hampshire UK, pp. 335-351 (2010)

15. Redden, E.S., Pettitt, R.A., Carstens, C.B., Elliott, L.R.: Scalability of robotic displays: Display size Investigation. Tech. report (ARL-TR-4456), ARL, APG, MD (2008)

16. Oron-Gilad, T., Redden, E.S., Minkov, Y.: Scalable OCUs for the Dismounted Soldier: Utilizing Unmanned Vehicles - A Field Study. J. Cog. Eng. \& Decision Making (Special Issue on Improving Human-Robot Interaction) (in press)

17. Redden, E.S., Pettitt, R.A., Carstens, C.B., Elliott, L.R., Rudnick, D.: Scaling Robotic Displays: Visual and Multimodal Options for Navigation by Dismounted Soldiers. Tech. report (ARL-TR-4708), ARL, APG, MD (2009)

18. Redden, E.S., Elliott, L.R., Pettitt, R.A., Carstens, C.B.: A Tactile Option to Reduce Robot Controller Size. J. Multimodal User Interfaces 2, 205-216 (2009)

19. Pettitt, R.A., Redden, E.S., Carstens, C.B.: Scalability of Robotic Controllers: An Evaluation of Controller Options. Tech. report (ARL-TR-4457), ARL, APG, MD (2008)

20. Pettitt, R., Redden, E., Carstens, C., Elliott, L.: Scalability of Robotic Controllers: Speechbased Robotic Controller Evaluation. Tech. report (ARL-TR-4858), ARL, APG, MD (2009)

21. Pettitt, R., Redden, E.S., Fung, T.: Scalbility of Robotic Controllers: An Evaluation of Controller Options, Experiment II. Tech. report. ARL, APG, MD (in review)

22. Elliott, L.R., Jansen, C., Redden, E.S., Pettitt, R.: Robotic Telepresence: Perception. Performance, and User Experience (in review)

23. Pettitt, R., Redden, E.S., Pacis, E., Carstens, C.B.: Scalability of Robotic Controllers: Effects of Progressive Levels of Autonomy on Robotic Reconnaissance Tasks. Tech. report (ARL-TR- 5258), ARL, APG, MD (2010) 